\title{
VEGETATION OF THE DOLINES IN MECSEK MOUNTAINS (SOUTH HUNGARY) IN RELATION TO THE LOCAL PLANT COMMUNITIES
}

\author{
VEGETACIJA V KRAŠKIH DEPRESIJAH IN NJIHOVA POVEZAVA \\ Z LOKALNIMI RASTLINSKIMI ZDRUŽBAMI, PRIMER \\ IZ GOROVJA MECSEK (MADŽARSKA)
}

\author{
Zoltán BÁTORI ${ }^{1}$, János CSIKY ${ }^{2}$, László ERDÖS², Tamás MORSCHHAUSER ${ }^{2}$, Péter TÖRÖK ${ }^{3}$ \\ \& László KÖRMÖCZI ${ }^{1}$
}

\begin{abstract}
UDC $630 * 1(493-13): 574$

Zoltán Bátori, János Csiky, László Erdős, Tamás Morschhauser, Péter Török \& László Körmöczi: Vegetation of the dolines in Mecsek mountains (South Hungary) in relation to the local plant communities

This paper deals with the forest vegetation of the lower part of the dolines in Mecsek Mts. (South Hungary). In order to characterize this vegetation type, samples were compared to the 6 plant communities occurring in the neighbourhood of the dolines. Considering the vegetation texture and species composition, the vegetation of the dolines resembles mainly the extrazonal beechwoods (Helleboro odori-Fagetum) and local ravine forests (Scutellario altissimae-Aceretum) that preserve several mountain, subatlantic relict species in this area. Our study revealed that the plant communities characteristic of the karst surface of Western Mecsek are arranged along a moisture and nutrient gradient. In this system, the habitat conditions of the dolines are similar to those of the beech forests and the local ravine forests, fresh and relatively rich in nutrients. In the karst, dominated by oak-hornbeam and beech forests, effects of the thermal inversion are the most spectacular where beech forests follow turkey oak-sessile oak forests and oak-hornbeam forests on the lower part of the doline slopes. The described vegetation type of these depressions is developed by edafic factors; its identification as a separate association is not supported by the analyses.
\end{abstract}

Keywords: doline, vegetation, diagnostic species, habitat conditions, ecological indicator values.
Izvleček

UDK $630 * 1(493-13): 574$

Zoltán Bátori, János Csiky, László Erdös, Tamás Morschhauser, Péter Török \& László Körmöczi: Vegetacija v kraških depresijah in njihova povezava z lokalnimi rastlinskimi združbami, primer iz gorovja Mecsek (Madžarska)

V članku obravnavamo gozdno vegetacijo spodnjega dela kraških depresij v gorovju Mescek (južna Madžarska). Da bi ovrednotili tip vegetacije, smo vzorce primerjali s šestimi rastlinskimi združbami v soseščini vrtač. Vegatacijska tekstura in vrstna sestava spominja na ekstraconalne bukove gozdove (Helleboro odori-Fagetum) in lokalne ravninske gozdove ( $\mathrm{Scu}$ tellario altissimae-Aceretum), v katerih je ohranjenih več gorskih subatlantskih reliktnih vrst tega območja. Naša raziskava je pokazala, da so rastlinske združbe na kraškem površju zahodnega dela gorovja Mecsek nanizane vzdolž gradientov vlage in hranil. V tem sistemu so habitatni pogoji v dolinah podobni pogojem $\mathrm{v}$ bukovih gozdovih in lokalnih ravninskim gozdovom, kjer so tla relativno bogata s hranili in vlažna. Na krasu, kjer prevladujejo hrastovo - gabrovi in bukovi gozdovi so učinki toplotne inverzije še posebej vidni. Tam bukovi gozdovi v spodnjih delih pobočij dolin sledijo cerovo - gradnovim (Quercus cerris - Q. petraea) in hrastovo - gabrovim združbam. $\mathrm{Na}$ tip vegetacije vplivajo predvsem talni faktorji, pri čemer naše analize kažejo, da ne gre za novo asociacijo.

Ključne besede: vrtače, doline, vegetacija, diagnostične vrste, habitatni pogoji, vrednosti ekoloških indikatorjev.

\footnotetext{
${ }^{1}$ University of Szeged, Department of Ecology, e-mail:Zbatory@gmail.com, kormoczi@bio.u-szeged.hu

${ }^{2}$ University of Pécs, Department of Plant Taxonomy and Geobotany, e-mail:[Moon@ttk.pte.hu, erdosl@gamma.ttk.pte.hu, morsi@gamma.ttk.pte.hu

${ }^{3}$ University of Debrecen, Department of Ecology, e-mail:[molinia@gmail.com

Received/Prejeto: 30.04.2009
} 


\section{INTRODUCTION}

Dolines are small to large sized closed depressions, from a few meters to a few hundred meters in diameter and depth, formed by water infiltration (Veress 2004). Previously, they were considered exclusively as collapse forms, but nowadays the process of doline formation is explained primarily by the change of soil activity on the bedrock (Jakucs 1980). Due to the special morphological, climati$\mathrm{cal}$ and vegetational peculiarities, dolines have become a centre of interest. Several researchers have investigated the special microclimate and the thermal inversion of the dolines (Bacsó \& Zólyomi 1934; Polli 1961, 1984; Futó 1962; Wagner 1963; Bárány 1967; Lehmann 1970; Jakucs 1971; Boros \& Bárány 1975; Bárány-Kevei 1999). Most of these studies also refer to the microclimatical differences between the slopes with a different exposure. The relationship between the thermal inversion and the vegetation was published, among others, by Beck v. Mannagetta (1906), Boros (1935), Morton (1936), Geiger (1950), Grom (1959), Jakucs (1961), Lausi (1964), Sauli (1972), Favretto and Poldini (1985), Kranjc (1997), Pericin and Hürlimann (2001), Borhidi (2002), Polli (2004).

Due to the special climatical conditions of the dolines and sinkholes, they often serve as habitats of rare and valuable species (Budai 1913; Yannitsaros et al. 1996; Tan et al. 1997; Vojtkó 1997; Varga et al. 2000; Bátori et al. 2006; Virók \& Farkas 2008), among which we can also find endemisms (Egli et al. 1990; Egli 1991; Brullo \& Giusso del Galdo 2001; Søndergaard \& Egli 2006). In the case of climate changes, the dolines and sinkholes may serve as shelters and their vegetation is often more ancient than the neighbouring vegetation (Jakucs 1952). Because of their ability to preserve the relicts (Bartha 1933; Horvat 1953; Atalay 2006), dolines have an important role in vegetation history.
A major part of Western Mecsek Mountains (South Hungary) is made up of red and gray Permic to lower Triassic sandstone and Triassic limestone. On the $30 \mathrm{~km}^{2}$ karstic surface nearby Abaliget, Mánfa, Orfü and Pécs, more than 2.200 dolines can be found. 1.702 of these dolines have a very small size ( $\mathrm{d}<20 \mathrm{~m}$; Rónaki 1972). The diameter of the largest one is more than $200 \mathrm{~m}$, its depth exceeds $25 \mathrm{~m}$ (Lovász 1971). The formation of these depressions started during the Pleistocene era and it is still intensive due also to the woodland and the abundant precipitation that exceeds $700 \mathrm{~mm}$ per year.

The oak-hornbeam woods (Asperulo taurinae-Carpinetum) and extrazonal beechwoods (Helleboro odoriFagetum) are the dominant plant communities on the plateaus and slopes between the dolines. Near to these forms, fragments of turkey oak-sessile oak forests (Potentillo micranthae-Quercetum dalechampii) and rock forests (Tilio tomentosae-Fraxinetum orni) also occur. In the deep valleys sorrounding the karstic surfaces there are ravine forests (Scutellario altissimae-Aceretum). Alder forests (Carici pendulae-Alnetum) can be found in sections of the valleys where streams meander in the deep alluvial deposit. The coenological surveys of these communities were published by Horvát $(1956,1958$, 1959, 1972), Kevey (1997), Kevey and Borhidi (1998) and Kevey and Baranyi (2002). The vegetation map of the smaller part of this area has been completed recently (Morschhauser et al. 2000).

The aim of this study was to analyse the species composition and vegetation texture in the dolines of Mecsek Mts. with the Braun-Blanquet method. Moreover, we used species indicator values in order to reveal the habitat conditions in the karst depressions.

\section{MATERIAL AND METHODS}

Phytosociological relevés were made in the lower part of the larger dolines $(\mathrm{d}>50 \mathrm{~m})$ in Western Mecsek applying the Central European method (Braun-Blanquet 1928). Relevés included the bottom of the dolines or part of them; their size is $400 \mathrm{~m}^{2}$. Due to their funnel-shape they show simultaneously different kinds of expositions and slopes, thus in the analytical table (Tab. 2) only the main ones are presented. Each vegetation relevé was recorded twice: during the spring and the summer aspects. We arranged the species in the table into syntaxonomical groups according to Kevey and Hirmann (2002).
Our 20 relevés were compared to the 120 relevés made by Kevey (1997), Kevey and Borhidi (1998) and Kevey and Baranyi (2002) from the surrounding vegetation of the dolines (6 plant associations).

The relationships among the species composition of the relevés were analysed with standard multivariate statistical methods (Pielou 1984; Podani 1994). Presence-absence (binary) datasets were analysed using Detrended Correspondence Analysis (DCA) ordination (Hill \& Gauch 1980), which is a common method for indirect gradient analysis. DCA results in an n-dimen- 
sional distribution pattern of objects and the rank of the dimensions. In the ordination scatter plot (Fig. 1; twodimensional hyperspace with the two most important dimensions or axes from the $\mathrm{n}$-dimension), similar relevés are close to each other and less similar relevés are further from each other, thus the distribution of the objects refers to the gradient phenomenon of the background factors. We used the program CANOCO 4.5 (Ter Braak \& Šmilauer 2002) for ordination.

Habitat conditions were characterized by the relative ecological indicator values (TWRN) built on the Ellenberg system and adapted to the Hungarian flora by Borhidi (1993). Distributions of indicator values were calculated for each relevé and vegetation unit concerning presence-absence and cover (weighted) data. In the case of cover data only the herb layer was considered.
When determining the diagnostic (differential) species, we used the method based on fidelity measurements (Chytrý et al. 2002; Tichý \& Chytrý 2006). The phi coefficient $(\Phi)$ was defined with the JUICE 7.0.25 program (Tichý 2002). This coefficient ranges from -1 to 1 , but for convenience, it is multiplied by 100 in the program. The highest phi value of 1 is achieved if the species occurs in all relevés of the target vegetation unit and is absent elsewhere. The species having a high fidelity $(\Phi>0,45)$ and at the same time belonging to only one vegetation unit were considered diagnostical.

Plant community names were used according to Borhidi (2003), and plant species names according to Simon (2000).

\section{RESULTS}

\section{FLORISTICAL CHARACTERISTICS AND DIAGNOSTIC SPECIES}

The DCA shows remarkable similarities among the relevés made in the lower part of the dolines in Western Mecsek. Considering the species composition, their vegetation resembles mainly the beechwoods and ravine forests, less the oak-hornbeam woods and alder forests. There is little resemblance between the vegetation of turkey oak-sessile oak forests, rock forests and dolines (Fig. 1).

The vegetation of the dolines is dominated by mesophilous forest plants (Fagetalia), but deciduous forests elements (Querco-Fagea), Illyrian beechwood elements (Aremonio-Fagion) and indifferent species also play an important role in this vegetation unit. In dolines, Helleboro odori-Fagetum and Scutellario altissimae-Aceretum stands there are several subatlantic relict and mountain species. Some of them (e.g. Dryopteris affinis, Dryopteris dilatata, Dryopteris expansa, Stachys alpina) occur both in dolines, beechwoods and ravine forests, while others (e.g. Actaea spicata, Aruncus dioicus, Lunaria rediviva, Silene dioica) primarily in ravine forests. Due to the cool and humid microclimate of the dolines, they may play a considerable role in the future in the preservation of these species.

Taking the above-mentioned vegetation units as the basis of the comparison, there are 4 diagnostic species for the dolines, 36 for the turkey oak-sessile oak forests, 8 for the rock forests, 3 for the oak-hornbeam forests, 7 for the ravine forests, 34 for the alder forests, but in this case, beechwoods do not have diagnostic species (Tab.
3). Diagnostic species of the dolines are Athyrium filixfemina, Paris quadrifolia, (Fagetalia), Atropa bella-donna (Atropion bella-donnae), Dryopteris carthusiana (Alnetea glutinosae).

Comparing the vegetation of the dolines only to the most similar beechwoods and ravine forests, there are 20 diagnostic species between the dolines and the beechwoods, and 41 between the dolines and the ravine forests. In this comparison, Athyrium filix-femina, Circaea lutetiana, Dryopteris filix-mas, Paris quadrifolia, Stachys sylvatica (Fagetalia), Chrysosplenium alternifolium (Alno-Padion), Polystichum aculeatum (Tilio-Acerion), Galium aparine, Urtica dioica (indifferent), Atropa belladonna, Dryopteris carthusiana are diagnostic in dolines, while Geranium robertianum (Querco-Fagea), Aegopodium podagraria, Hepatica nobilis, Lathyrus vernus, Prunus avium, Tilia platyphyllos (Fagetalia), Lathyrus venetus, Ruscus aculeatus, Tamus communis (Aremonio-Fagion) in beechwoods.

Only 4 species occur in the dolines (Athyrium filix-femina, Atropa bella-donna, Dryopteris carthusiana, Paris quadrifolia) that can be considered differential species compared to the ravine forests, while in these forests there are 37 species that can not be found in the dolines, or just occasionally. Most of these are Querco-Fagea (Campanula rapunculoides, Clematis vitalba, Corylus avellana, Crataegus monogyna, Crataegus laevigata, Euonymus verrucosus, Geranium robertianum, Ligustrum vulgare, Staphylea pinnata, Tilia platyphyllos), Fagetalia (Aconitum vulparia, Aegopodium podagraria, Cardamine enneaphyllos, Cerastium sylvaticum, Corydalis cava, 


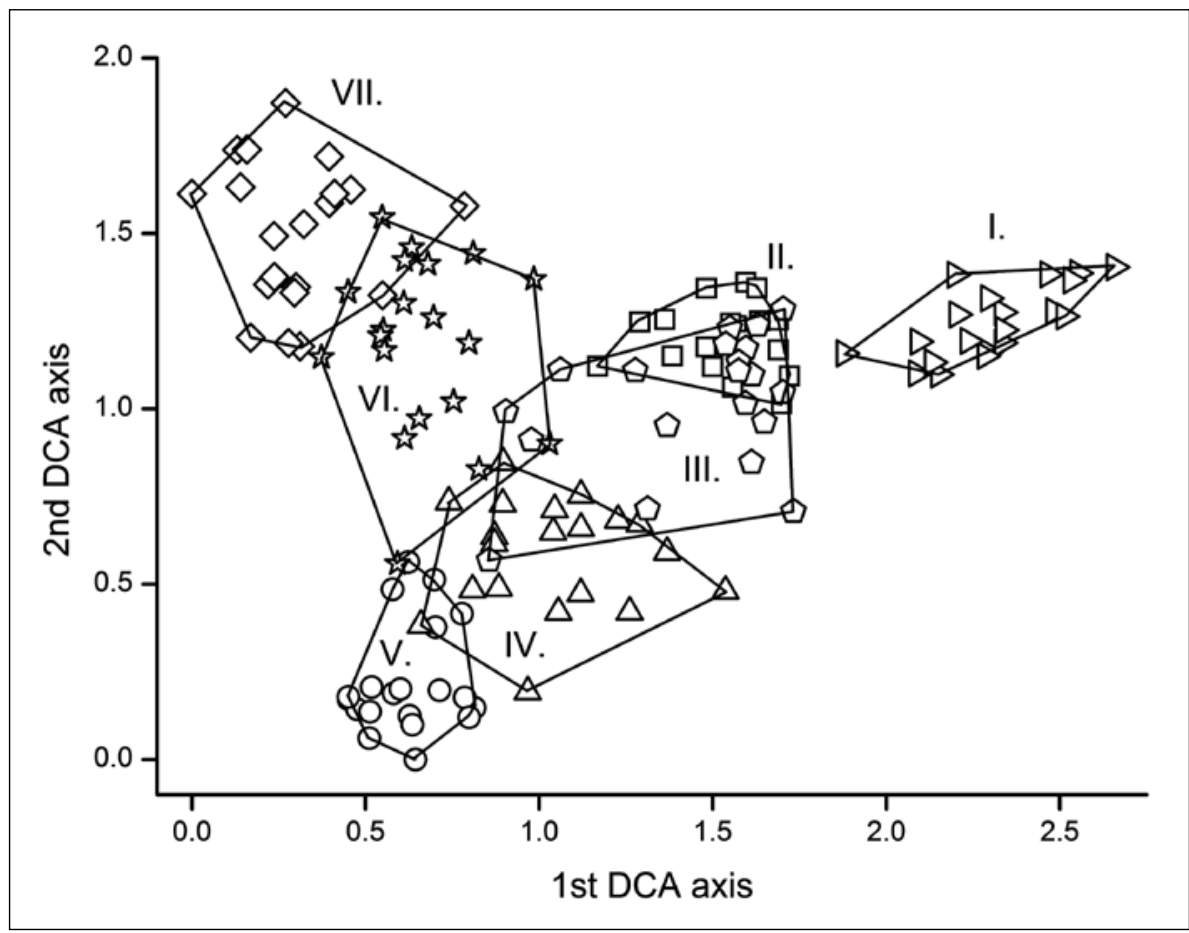

Fig. 1: DCA ordination diagram of 140 relevés (7 vegetation units) of the study area. Notations: I: Potentillo micranthae-Quercetum dalechampii, II: Tilio tomentosae-Fraxinetum orni, III: Asperulo taurinae-Carpinetum, IV: Helleboro odori-Fagetum, V: Vegetation of the dolines, VI: Scutellario altissimae-Aceretum, VII: Carici pendulae-Alnetum. Eigenvalues for the $1^{\text {st }}$ and $2^{\text {nd }}$ axis were 0.309 and 0.103 , respectively.

Gagea lutea, Geranium phaeum, Isopyrum thalictroides, Knautia drymeia, Lathyrus vernus, Oxalis acetosella, Ranunculus lanuginosus, Salvia glutinosa), or Tilio-Acerion (Asplenium scolopendrium, Cystopteris fragilis, Lunaria rediviva, Silene dioica) species. Alliaria petiolata (GalioAlliarion), Asplenium trichomanes (Asplenio-Festucion pallentis), Cardamine amara (Cardamini-Montion), Carex remota (Alnetea glutinosae), Polystichum setiferum (Aremonio-Fagion), Chelidonium majus, Ranunculus repens, Stellaria media (indifferent), Robinia pseudo-acacia (adventiva) and Chrysosplenium alternifolium are also diagnostic in ravine forests.

\section{STRUCTURAL CHARACTERISTICS}

The structure of the canopy in the dolines is very similar to that of the ravine forests. Upper canopy cover varies between 55 and $90 \%$, while the canopy height varies between 23 and $30 \mathrm{~m}$. Upper canopy is primarily composed of Acer campestre, Acer platanoides, Acer pseudoplatanus, Carpinus betulus, Fagus sylvatica and Tilia tomentosa. Some other species (Fraxinus excelsior, Populus tremula, Quercus petraea, Tilia cordata, Ulmus glabra) also play an important role, while other trees (e.g. Quercus cerris) occur sporadically. Younger trees form a lower canopy with $0-20 \%$ cover and 7-17 m height.

The shrub layer is primarily composed of young trees of the canopy layer, with a cover of $0-60 \%$ and 1-5 m in height. While Sambucus nigra is also typical in this level, others (e.g. Prunus avium, Tilia platyphyllos) occur sporadically.

The herb layer is mostly well developed with a cover varying between 60-100\%, and an average height of $25-70 \mathrm{~cm}$. Frequent species include: Allium ursinum, Athyrium filix-femina, Carex pilosa, Carex sylvatica, Circaea lutetiana, Dryopteris carthusiana, Dryopteris filixmas, Galeobdolon luteum s.l., Galium odoratum, Hedera helix, Helleborus odorus, Mercurialis perennis, Moehringia trinervia, Paris quadrifolia, Polygonatum multiflorum, Polystichum aculeatum, Pulmonaria officinalis, Rubus hirtus agg., Ruscus hypoglossum, Stachys sylvatica, Veronica montana, Viola reichenbachiana. During the springtime Anemone ranunculoides, Arum maculatum s.str., Cardamine bulbifera, Ficaria verna, Galanthus nivalis are also common.

In the herb layer of deeper dolines, the presence of fern species (Athyrium filix-femina, Dryopteris affinis, Dryopteris carthusiana, Dryopteris dilatata, cf. Dryopteris expansa, Dryopteris filix-mas, Polystichum aculeatum, Polystichum setiferum) and wet woodland species (e.g. Chrysosplenium alternifolium, Urtica dioica) indicates a cool and humid microclimate, and high soil humidity. Because of the open canopy, Atropa bella-donna appears as well in the lower part of the dolines. The species of the herb layer also often occur on the decayed, mossy trees fallen into the bottom of the dolines.

\section{HABITAT CONDITIONS BASED ON ECOLOGICAL INDICATOR VALUES}

Habitat conditions of the compared vegetation units can be characterized by the ecological indicator values. The 7 vegetation units show differences according to temperature $(T)$, moisture supply $(W)$, soil reaction $(R)$, and 
Tab. 1: $W$ and $N$ indicator spectra of the vegetation of the study area (maximum values are set in bold).

\begin{tabular}{|c|c|c|c|c|c|c|c|}
\hline $\begin{array}{l}\text { Binary data } \\
(\%)\end{array}$ & $\begin{array}{r}\text { I. } \\
\text { PmQd }\end{array}$ & $\begin{array}{r}\text { II. } \\
\text { TtFo }\end{array}$ & $\begin{array}{l}\text { III. } \\
\text { AtC }\end{array}$ & $\begin{array}{r}\text { IV. } \\
\text { HoF }\end{array}$ & $\begin{array}{r}\text { V. } \\
\text { Dolines }\end{array}$ & $\begin{array}{r}\text { VI. } \\
\text { SaA }\end{array}$ & $\begin{array}{r}\text { VII. } \\
\text { CpA }\end{array}$ \\
\hline W2 & 0.32 & 0.11 & 0.00 & 0.00 & 0.00 & 0.00 & 0.12 \\
\hline W3 & 9.51 & 4.96 & 1.28 & 0.42 & 0.22 & 1.72 & 0.90 \\
\hline W4 & 19.68 & 10.66 & 11.25 & 4.68 & 2.63 & 5.63 & 4.87 \\
\hline W5 & 52.04 & 45.07 & 45.75 & 41.37 & 36.86 & 31.57 & 24.59 \\
\hline W6 & 14.56 & 31.97 & 35.31 & 43.97 & 43.86 & 40.44 & 34.58 \\
\hline W7 & 3.88 & 7.24 & 6.33 & 9.56 & 15.88 & 16.28 & 18.88 \\
\hline W8 & 0.00 & 0.00 & 0.07 & 0.00 & 0.22 & 2.51 & 7.40 \\
\hline W9 & 0.00 & 0.00 & 0.00 & 0.00 & 0.33 & 1.79 & 7.70 \\
\hline W10 & 0.00 & 0.00 & 0.00 & 0.00 & 0.00 & 0.07 & 0.48 \\
\hline W11 & 0.00 & 0.00 & 0.00 & 0.00 & 0.00 & 0.00 & 0.48 \\
\hline N1 & 0.39 & 0.00 & 0.00 & 0.00 & 0.00 & 0.00 & 0.00 \\
\hline N2 & 5.70 & 1.25 & 1.68 & 1.46 & 0.55 & 0.40 & 0.96 \\
\hline N3 & 16.65 & 7.24 & 4.18 & 0.94 & 2.08 & 1.85 & 2.83 \\
\hline N4 & 25.45 & 20.74 & 17.45 & 14.35 & 9.08 & 15.68 & 11.85 \\
\hline N5 & 25.65 & 25.75 & 31.40 & 31.70 & 26.48 & 22.83 & 21.05 \\
\hline N6 & 12.50 & 14.47 & 16.71 & 19.75 & 23.63 & 16.41 & 14.25 \\
\hline N7 & 8.48 & 20.28 & 19.14 & 24.01 & 29.43 & 30.05 & 30.61 \\
\hline N8 & 3.82 & 9.23 & 8.49 & 7.59 & 6.89 & 10.99 & 14.07 \\
\hline N9 & 1.36 & 1.03 & 0.94 & 0.21 & 1.86 & 1.79 & 4.39 \\
\hline $\begin{array}{l}\text { Cover data } \\
(\%)\end{array}$ & $\begin{array}{r}\text { I. } \\
\text { PmQd }\end{array}$ & $\begin{array}{r}\text { II. } \\
\text { TtFo }\end{array}$ & $\begin{array}{l}\text { III. } \\
\text { AtC }\end{array}$ & $\begin{array}{r}\text { IV. } \\
\text { HoF }\end{array}$ & $\begin{array}{r}\mathrm{V} . \\
\text { Dolines }\end{array}$ & $\begin{array}{r}\text { VI. } \\
\text { SaA }\end{array}$ & $\begin{array}{l}\text { VII. } \\
\text { CpA }\end{array}$ \\
\hline W2 & 0.10 & 0.06 & 0.00 & 0.00 & 0.00 & 0.00 & 0.03 \\
\hline W3 & 2.28 & 2.37 & 0.08 & 0.02 & 0.00 & 0.36 & 0.22 \\
\hline W4 & 19.37 & 11.29 & 3.37 & 1.03 & 0.54 & 1.28 & 0.44 \\
\hline W5 & 71.90 & 32.65 & 28.84 & 26.92 & 15.60 & 12.65 & 8.52 \\
\hline W6 & 4.89 & 48.83 & 63.44 & 64.21 & 77.06 & 68.22 & 67.68 \\
\hline W7 & 1.37 & 3.23 & 3.89 & 7.40 & 6.72 & 9.83 & 18.85 \\
\hline W8 & 0.00 & 0.00 & 0.02 & 0.00 & 0.03 & 0.68 & 2.55 \\
\hline W9 & 0.00 & 0.00 & 0.00 & 0.00 & 0.05 & 0.56 & 1.24 \\
\hline W10 & 0.00 & 0.00 & 0.00 & 0.00 & 0.00 & 0.02 & 0.11 \\
\hline W11 & 0.00 & 0.00 & 0.00 & 0.00 & 0.00 & 0.00 & 0.11 \\
\hline N1 & 0.12 & 0.00 & 0.00 & 0.00 & 0.00 & 0.00 & 0.00 \\
\hline N2 & 1.37 & 0.15 & 0.12 & 0.05 & 0.00 & 0.02 & 0.23 \\
\hline N3 & 7.90 & 3.26 & 0.65 & 0.14 & 0.37 & 0.65 & 0.53 \\
\hline N4 & 55.29 & 21.39 & 14.68 & 7.93 & 1.86 & 7.86 & 4.17 \\
\hline N5 & 24.66 & 18.19 & 17.78 & 32.19 & 29.71 & 21.23 & 23.27 \\
\hline N6 & 4.40 & 9.93 & 6.59 & 7.29 & 13.35 & 6.68 & 3.51 \\
\hline N7 & 4.11 & 31.61 & 10.62 & 16.38 & 13.23 & 21.13 & 19.30 \\
\hline N8 & 1.64 & 13.36 & 48.96 & 35.58 & 40.61 & 35.56 & 45.66 \\
\hline N9 & 0.44 & 0.53 & 0.23 & 0.05 & 0.86 & 0.49 & 3.09 \\
\hline
\end{tabular}

nitrogen supply $(\mathrm{N})$. The biggest differences are shown in the case of $\mathrm{W}$ and $\mathrm{N}$ values, which can be successfully applied in local scale (Salamon-Albert \& Morschhauser 2002) as well.

The use of $\mathrm{W}$ values (Tab. 1) with binary data shows that the proportion of W5 (plants of semi-humid habitats, under intermediate conditions) species is the highest in the $\mathrm{W}$ indicator spectra of turkey oak-sessile oak forests, oak-hornbeam forests and rock forests, while in the other units the rate of W6 (plants of fresh soils) species is the most dominant. The proportion of W3 (xerotolerants, but eventually occurring on fresh soils), W4 (plants of semi-dry habitats) and W6 species in turkey oak-sessile oak forests is also important, and this is the same in the case of W5 and W7 (plants of moist soils not drying out and well aerated) species in ravine forests and W5, W7, W8 (plants of moist soils tolerating short floods) and W9 (plants of wet, not well aerated soils) species in alder forests. Vegetation units are also divided by the weighted data, showing the maximum at $\mathrm{W} 5$ in turkey oak-sessile oak forests, and in the rest of the units the maximum is found at W6. The proportion of W4 species is also high in turkey oak-sessile oak forests, while W5 species are frequent in oak-hornbeam, rock, and beech forests, and W7 species in alder forests. The $\mathrm{W}$ spectra of the dolines resemble mostly the spectra of beech forests and ravine forests at the maximum of W6. 
Tab. 2: Analytical table of the doline vegetation of the Western Mecsek.

\begin{tabular}{lllllllllllllllllllllll}
1 & 2 & 3 & 4 & 5 & 6 & 7 & 8 & 9 & 10 & 11 & 12 & 13 & 14 & 15 & 16 & 17 & 18 & 19 & 20 & A-D & K & $\%$ \\
\hline
\end{tabular}

\section{Phragmitetea}

Eupatorium cannabinum

Lycopus europaeus

Solanum dulcamara

\section{Galio-Alliarion}

Alliaria petiolata

\section{Calystegion sepium}

Lamium maculatum

Bidentetea (incl. Bidentetalia)

Persicaria mitis

C

Atropion bella-donnae

Atropa bella-donna

\section{Querco-Fagea}

Acer campestre

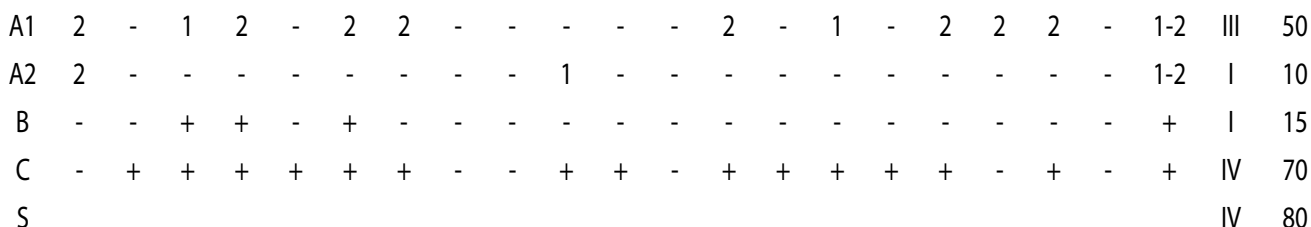

Ajuga reptans

Brachypodium sylvaticum

Bromus ramosus agg.

Carex divulsa

Clematis vitalba

Cornus sanguinea

Crataegus laevigata

Dactylis polygama

Euonymus europaeus

Ficaria verna

Fragaria vesca

Fraxinus excelsior

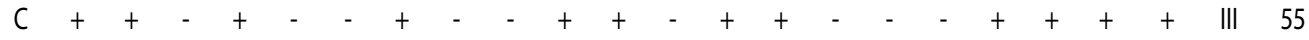

Geranium robertianum Geum urbanum

Heracleum sphondylium Hypericum hirsutum

Melica uniflora

Mycelis muralis

Polygonatum multiflorum Populus tremula 


\begin{tabular}{|c|c|c|c|c|c|c|c|c|c|c|c|c|c|c|c|c|c|c|c|c|c|c|c|c|}
\hline & & 1 & 2 & 3 & 4 & 5 & 6 & 7 & 8 & 9 & 10 & 11 & 12 & 13 & 14 & 15 & 16 & 17 & 18 & 19 & 20 & $A-D$ & $\mathrm{~K}$ & $\%$ \\
\hline \multirow[t]{3}{*}{ Quercus petraea } & $\mathrm{A} 1$ & - & - & - & - & 1 & - & - & - & - & - & - & - & 2 & - & - & - & - & - & - & - & $1-2$ & I & 10 \\
\hline & $C$ & - & + & - & - & - & - & - & - & - & - & - & - & - & + & + & + & - & - & - & + & + & $\|$ & 25 \\
\hline & $S$ & & & & & & & & & & & & & & & & & & & & & & $\|$ & 35 \\
\hline Scrophularia nodosa & $C$ & - & + & + & - & - & + & - & - & - & - & - & - & - & - & - & - & - & + & - & + & + & $\|$ & 25 \\
\hline Staphylea pinnata & C & - & - & - & - & - & - & - & - & - & - & - & - & - & - & - & + & - & - & - & - & + & I & 5 \\
\hline Stellaria holostea & $\mathrm{C}$ & - & - & - & - & - & - & - & + & - & + & + & - & + & - & + & + & - & - & - & - & + & $\|$ & 30 \\
\hline Symphytum tuberosum & C & + & - & + & - & - & - & - & - & - & - & + & - & - & - & - & - & - & - & - & - & + & I & 15 \\
\hline \multirow[t]{4}{*}{ Tilia cordata } & $\mathrm{A} 1$ & - & 1 & - & - & - & 1 & - & 2 & - & - & - & 2 & - & - & - & - & - & - & - & - & $1-2$ & I & 20 \\
\hline & B & - & 2 & - & - & - & 1 & - & 2 & - & - & - & 2 & - & - & - & + & - & - & + & + & +-2 & $\|$ & 35 \\
\hline & $C$ & - & 1 & - & - & - & - & - & + & - & + & + & - & - & - & + & - & - & - & - & - & +-1 & $\|$ & 25 \\
\hline & $S$ & & & & & & & & & & & & & & & & & & & & & & III & 50 \\
\hline Veronica chamaedrys & C & - & + & + & - & + & - & - & - & - & + & + & - & + & - & - & - & - & - & - & + & + & $\|$ & 35 \\
\hline Veronica hederifolia & C & - & - & - & - & - & - & - & - & - & - & - & - & - & - & - & - & + & - & + & - & + & I & 10 \\
\hline Viola alba & $\mathrm{C}$ & - & - & - & - & - & - & - & - & - & - & - & - & - & - & - & - & + & - & - & - & + & । & 5 \\
\hline
\end{tabular}

\section{Dryopteris carthusiana Dryopteris dilatata Dryopteris expansa}

Acer platanoides

Acer pseudoplatanus

Aconitum vulparia

Actaea spicata

Aegopodium podagraria

Allium ursinum

Anemone ranunculoides

Arum maculatum s.str.

Asarum europaeum

Athyrium filix-femina

Cardamine bulbifera

Cardamine enneaphyllos

Cardamine impatiens

Carex digitata

Carex pilosa

Carex sylvatica

Carpinus betulus

\section{Alnetea glutinosae}

$\begin{array}{llllll}\mathrm{C} & + & + & + & + & - \\ \mathrm{C} & + & - & - & + & + \\ \mathrm{C} & - & - & - & - & -\end{array}$

Carpino-Fagetea (incl. Fagetalia)

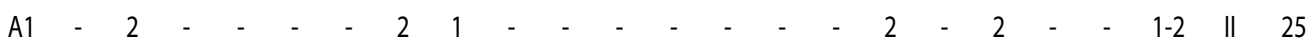

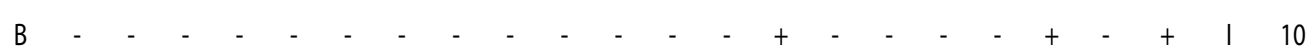

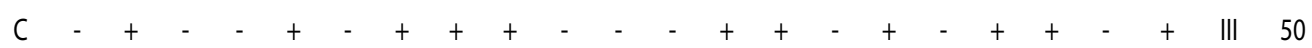

$S \quad$ III 50

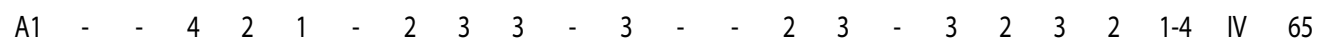

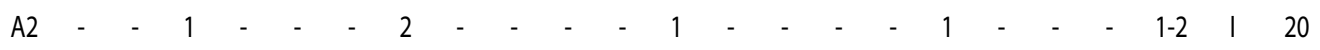

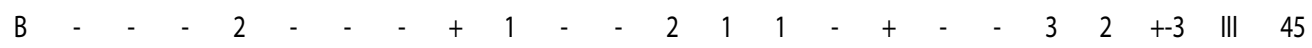

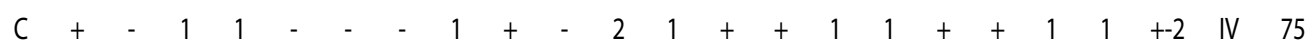

S $\quad$ V 85

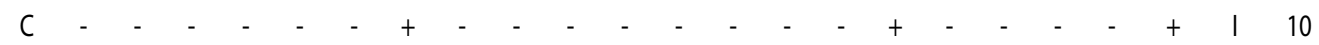

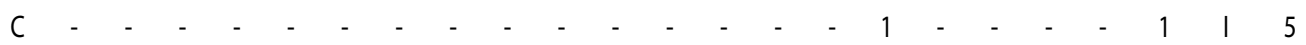

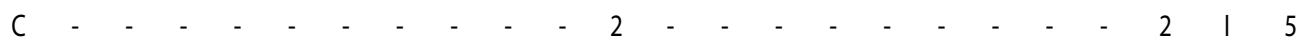

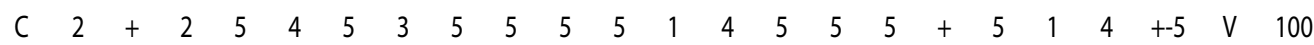

$\mathrm{C} 1++1+++++++++++++1-+1+-1 \vee 95$

$\mathrm{C}++++++++++++++++++1++t_{+}+1 \quad \mathrm{~V} 100$

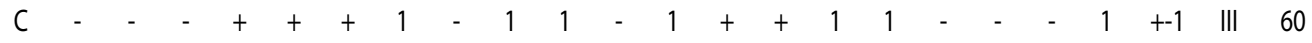

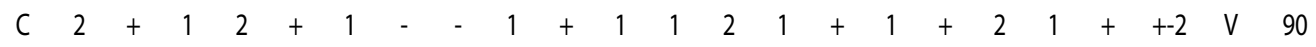

$\mathrm{C} 2+1+++1++++1++++2++1+2 \mathrm{~V} 100$

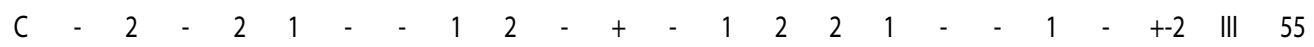

C - - + - + - - - - - - - + - - - - + - - + I 20

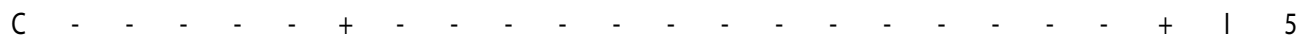

$\mathrm{C}-1+++1111+++11-++2+11+-2 \mathrm{~V} 90$

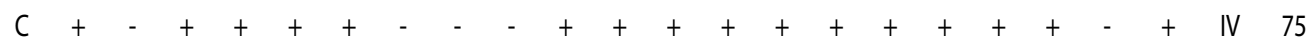

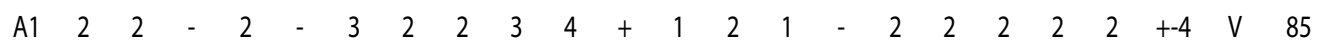

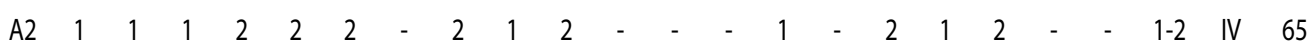

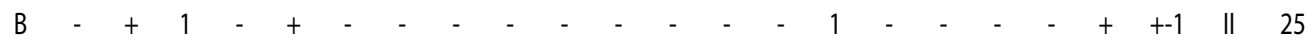

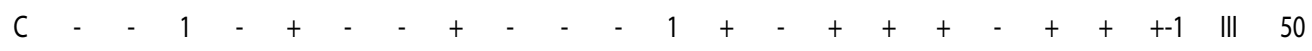




\begin{tabular}{|c|c|c|c|c|c|c|c|c|c|c|c|c|c|c|c|c|c|c|c|c|c|c|c|c|}
\hline & & 1 & 2 & 3 & 4 & 5 & 6 & 7 & 8 & 9 & 10 & 11 & 12 & 13 & 14 & 15 & 16 & 17 & 18 & 19 & 20 & $A-D$ & K & $\%$ \\
\hline Circaea lutetiana & $\mathrm{C}$ & + & + & + & + & 2 & + & + & + & + & + & + & 2 & 1 & 1 & + & + & - & + & 1 & 1 & +-2 & V & 95 \\
\hline Corydalis cava & $C$ & - & - & - & - & - & - & - & - & - & - & - & - & - & 1 & - & 1 & - & - & + & - & +-1 & I & 15 \\
\hline Dryopteris filix-mas & $\mathrm{C}$ & + & 1 & 1 & 1 & + & + & 1 & 1 & 1 & 1 & 1 & 1 & + & + & 1 & 2 & + & 1 & 1 & + & +-2 & V & 100 \\
\hline Epilobium montanum & $C$ & - & - & - & - & - & - & - & - & - & - & - & - & - & - & - & + & - & - & - & - & + & I & 5 \\
\hline Euphorbia amygdaloides & $\mathrm{C}$ & - & - & - & - & - & - & + & - & + & - & - & - & - & - & + & - & - & - & - & + & + & I & 20 \\
\hline \multirow[t]{5}{*}{ Fagus sylvatica } & $\mathrm{A} 1$ & 3 & 2 & - & - & 2 & 2 & - & - & 2 & - & 3 & 2 & 2 & 2 & 2 & - & 2 & - & 3 & 2 & $2-3$ & IV & 65 \\
\hline & $A 2$ & - & - & - & - & 1 & 1 & - & - & 1 & - & 1 & 1 & - & 1 & - & 1 & - & - & - & 1 & 1 & ॥ & 40 \\
\hline & $B$ & - & 1 & - & + & - & - & 1 & 1 & + & - & 1 & 2 & - & - & 1 & - & - & - & 1 & + & +-2 & III & 50 \\
\hline & $C$ & + & + & - & - & - & - & + & + & - & - & + & 1 & + & 1 & + & - & + & - & + & + & +-1 & III & 60 \\
\hline & $S$ & & & & & & & & & & & & & & & & & & & & & & V & 85 \\
\hline Festuca drymeja & $\mathrm{C}$ & - & - & - & - & - & - & - & - & - & - & - & - & + & - & + & + & - & - & - & - & + & I & 15 \\
\hline Gagea lutea & $\mathrm{C}$ & + & + & - & + & - & - & - & - & + & - & - & - & - & - & - & + & - & + & - & - & + & II & 30 \\
\hline Galanthus nivalis & $\mathrm{C}$ & - & + & + & 1 & 1 & + & 1 & 1 & + & + & + & 1 & + & + & + & + & + & + & + & 1 & +-1 & V & 95 \\
\hline Galeobdolon luteum s. I. & $\mathrm{C}$ & 2 & 2 & 3 & 3 & 3 & 2 & 3 & 3 & 2 & 3 & 3 & 2 & 2 & 4 & 4 & 4 & 2 & 4 & 3 & 3 & $2-4$ & V & 100 \\
\hline Galeopsis speciosa & $\mathrm{C}$ & + & - & + & - & - & - & - & - & - & - & + & - & - & - & - & - & - & - & - & - & + & I & 15 \\
\hline Galium odoratum & $\mathrm{C}$ & + & 2 & 1 & + & 1 & - & 1 & 1 & + & + & 1 & 1 & + & + & 1 & + & 1 & - & 1 & 1 & +-2 & V & 90 \\
\hline Geranium phaeum & $C$ & - & - & - & - & - & - & - & - & - & - & + & - & - & - & - & - & - & - & - & - & + & I & 5 \\
\hline \multirow[t]{3}{*}{ Hedera helix } & $\mathrm{A} 1$ & - & + & - & - & - & - & - & - & - & - & + & - & - & - & - & - & - & - & 1 & - & +-1 & I & 15 \\
\hline & $C$ & + & 2 & + & + & - & 1 & + & 1 & 1 & + & 1 & 1 & 1 & + & 1 & + & + & + & 1 & 1 & +-2 & V & 95 \\
\hline & $S$ & & & & & & & & & & & & & & & & & & & & & & V & 95 \\
\hline Hepatica nobilis & $\mathrm{C}$ & - & + & - & - & - & - & - & - & - & + & + & - & - & - & + & - & - & - & 1 & + & +-1 & ॥ & 30 \\
\hline Hordelymus europaeus & $\mathrm{C}$ & - & + & - & - & - & + & - & - & - & - & - & - & - & + & + & - & - & - & - & - & + & I & 20 \\
\hline Isopyrum thalictroides & $\mathrm{C}$ & - & + & - & + & - & - & + & - & + & - & + & - & - & - & + & - & - & - & - & + & + & ॥ & 35 \\
\hline Lathraea squamaria & $C$ & - & + & - & + & - & + & + & + & + & + & - & - & - & + & - & - & + & + & - & - & + & III & 50 \\
\hline Lathyrus vernus & $\mathrm{C}$ & - & - & - & + & - & - & - & - & - & - & + & - & - & - & - & - & - & - & - & - & + & I & 10 \\
\hline Lilium martagon & $C$ & - & - & - & - & - & - & - & - & - & - & + & - & - & - & - & - & - & - & - & - & + & I & 5 \\
\hline Mercurialis perennis & $C$ & - & 1 & - & 1 & - & 1 & 2 & 1 & 1 & + & 1 & 2 & + & + & 1 & + & - & + & 1 & 1 & +-2 & IV & 80 \\
\hline Milium effusum & $C$ & - & - & - & - & - & - & - & - & - & + & - & + & - & - & - & + & - & - & - & + & + & I & 20 \\
\hline Moehringia trinervia & $\mathrm{C}$ & + & - & + & + & + & + & + & - & - & + & + & + & + & - & + & + & - & + & - & + & + & IV & 70 \\
\hline Oxalis acetosella & $\mathrm{C}$ & + & + & 1 & + & + & + & 1 & 1 & + & 1 & 1 & - & - & - & - & - & - & - & - & - & +-1 & III & 55 \\
\hline Paris quadrifolia & $\mathrm{C}$ & + & + & 1 & - & + & - & + & + & + & - & + & + & - & + & + & + & - & - & + & + & +-1 & IV & 70 \\
\hline \multirow[t]{3}{*}{ Prunus avium } & B & - & - & - & - & - & - & - & - & - & - & - & - & - & - & - & - & - & - & + & - & + & 1 & 5 \\
\hline & $\mathrm{C}$ & - & + & - & - & - & - & - & - & - & - & - & - & - & - & - & - & + & - & + & - & + & I & 15 \\
\hline & $S$ & & & & & & & & & & & & & & & & & & & & & & 1 & 15 \\
\hline Pulmonaria officinalis & $\mathrm{C}$ & + & + & + & + & + & - & + & + & + & + & + & 1 & - & - & + & + & + & - & + & + & +-1 & IV & 80 \\
\hline Ranunculus lanuginosus & $\mathrm{C}$ & - & - & - & - & - & - & - & - & - & + & - & - & - & - & - & - & - & - & - & - & + & 1 & 5 \\
\hline Rubus hirtus agg. & $\mathrm{C}$ & - & 1 & 2 & + & 2 & 2 & + & 1 & 2 & 1 & 2 & + & 1 & + & 2 & + & 1 & 1 & 2 & 2 & +-2 & V & 95 \\
\hline Stachys alpina & $C$ & - & + & - & - & - & - & - & - & - & - & - & - & - & - & - & - & - & - & - & - & + & I & 5 \\
\hline Stachys sylvatica & $\mathrm{C}$ & + & - & + & + & + & + & - & + & + & - & 1 & + & + & + & + & + & - & - & - & + & +-1 & IV & 70 \\
\hline \multirow[t]{3}{*}{ Tilia platyphyllos } & B & - & - & - & - & - & - & - & - & - & - & - & - & 2 & - & - & - & - & - & - & - & 2 & 1 & 5 \\
\hline & $C$ & - & - & - & - & - & - & - & - & - & - & - & - & + & - & - & - & - & - & - & - & + & I & 5 \\
\hline & $S$ & & & & & & & & & & & & & & & & & & & & & & 1 & 5 \\
\hline \multirow[t]{4}{*}{ Ulmus glabra } & $\mathrm{A} 1$ & - & - & - & - & - & - & - & - & - & - & - & - & - & 1 & - & - & - & - & 2 & - & $1-2$ & 1 & 10 \\
\hline & $B$ & + & + & - & - & - & - & - & + & - & - & + & - & - & - & + & - & - & - & + & - & + & ॥ & 30 \\
\hline & $C$ & + & - & + & - & - & + & + & - & - & - & + & - & - & - & + & - & - & - & 1 & + & +-1 & ॥ & 40 \\
\hline & $S$ & & & & & & & & & & & & & & & & & & & & & & III & 55 \\
\hline Veronica montana & $\mathrm{C}$ & + & + & + & + & + & + & + & + & + & + & + & + & + & + & + & + & + & + & 1 & + & +-1 & V & 100 \\
\hline Viola reichenbachiana & $C$ & + & + & + & + & + & + & + & + & + & + & + & + & + & + & + & + & + & + & + & + & + & V & 100 \\
\hline
\end{tabular}




\begin{tabular}{|c|c|c|c|c|c|c|c|c|c|c|c|c|c|c|c|c|c|c|c|c|c|c|c|c|}
\hline & & 1 & 2 & 3 & 4 & 5 & 6 & 7 & 8 & 9 & 10 & 11 & 12 & 13 & 14 & 15 & 16 & 17 & 18 & 19 & 20 & $A-D$ & K & $\%$ \\
\hline \multicolumn{25}{|c|}{ Alno-Padion } \\
\hline Carexpendula & C & - & - & - & - & - & - & - & - & - & - & - & - & - & + & - & + & - & - & - & - & + & I & 10 \\
\hline $\begin{array}{l}\text { Chrysosplenium } \\
\text { alternifolium }\end{array}$ & C & - & - & - & 1 & - & - & - & - & + & + & + & + & 1 & 1 & - & - & - & + & - & + & +-1 & III & 45 \\
\hline Festuca gigantea & C & - & - & - & - & - & - & - & - & - & - & - & - & + & - & - & + & - & - & - & - & + & I & 10 \\
\hline Rumex sanguineus & $C$ & - & - & + & - & - & - & - & - & - & - & - & - & - & - & - & + & - & - & - & - & + & I & 10 \\
\hline \multicolumn{25}{|c|}{ Eu-Fagion } \\
\hline Dryopteris affinis & $C$ & - & - & - & - & - & - & - & - & - & - & - & + & - & - & - & + & + & + & - & - & + & I & 20 \\
\hline \multicolumn{25}{|c|}{ Tilio-Acerion } \\
\hline Polystichum aculeatum & $C$ & + & + & - & + & + & - & - & + & + & + & - & + & 1 & + & + & 1 & + & - & - & - & +-1 & IV & 65 \\
\hline \multicolumn{25}{|c|}{ Aremonio-Fagion } \\
\hline Aremonia agrimonoides & $C$ & - & - & - & - & - & - & - & - & - & - & - & + & - & - & - & + & - & - & - & - & + & I & 10 \\
\hline Helleborus odorus & $C$ & + & + & + & + & - & + & - & + & + & + & + & + & - & + & + & + & + & + & 1 & 1 & +-1 & V & 85 \\
\hline Polystichum setiferum & $\mathrm{C}$ & + & - & - & - & - & - & - & - & - & - & - & - & + & - & - & - & - & + & + & - & + & I & 20 \\
\hline Rosa arvensis & C & - & - & - & - & - & - & - & - & - & - & - & + & - & - & - & - & - & - & - & + & + & I & 10 \\
\hline Ruscus aculeatus & $C$ & + & 1 & - & - & - & - & - & - & + & - & - & + & + & - & + & - & - & - & + & - & +-1 & $\|$ & 35 \\
\hline Ruscus hypoglossum & $C$ & + & + & - & - & + & + & + & + & + & + & - & - & + & - & - & - & + & + & + & + & + & IV & 65 \\
\hline Tamus communis & $\mathrm{C}$ & - & - & - & - & - & + & - & - & - & - & - & - & - & - & - & - & + & - & - & - & + & I & 10 \\
\hline \multicolumn{25}{|c|}{ Quercetea pubescentis-petraeae } \\
\hline Fraxinus ornus & $\mathrm{C}$ & - & - & - & - & - & - & - & - & - & - & - & - & - & - & + & - & - & - & + & - & + & I & 10 \\
\hline \multirow{3}{*}{ Quercus cerris } & $\mathrm{A} 1$ & - & 2 & - & - & - & - & - & - & - & - & - & - & - & - & - & - & - & - & - & - & 2 & I & 5 \\
\hline & $C$ & - & - & + & + & - & + & + & - & - & + & - & - & - & - & + & - & + & - & - & - & + & II & 35 \\
\hline & $S$ & & & & & & & & & & & & & & & & & & & & & & ॥ & 40 \\
\hline \multicolumn{25}{|c|}{ Quercion farnetto } \\
\hline \multirow[t]{6}{*}{ Tilia tomentosa } & $\mathrm{A} 1$ & - & - & - & - & 1 & - & - & 2 & - & - & - & 3 & - & 2 & 2 & 3 & - & 2 & - & 2 & $1-3$ & $\|$ & 40 \\
\hline & A2 & - & - & - & - & - & - & - & - & - & - & - & - & 1 & - & - & - & - & - & - & - & 1 & I & 5 \\
\hline & B & - & 1 & - & 1 & 2 & 1 & - & + & - & - & + & 2 & 2 & 3 & 2 & 2 & - & + & - & 1 & +-3 & IV & 65 \\
\hline & C & - & 1 & + & + & 1 & - & + & + & + & - & - & 1 & + & + & 1 & 1 & - & - & + & + & +-1 & IV & 70 \\
\hline & $S$ & & & & & & & & & & & & & & & & & & & & & & V & 85 \\
\hline & & & & & & & & \multicolumn{17}{|c|}{ Indifferent } \\
\hline Galium aparine & C & - & - & + & - & + & - & - & - & - & + & + & - & - & + & + & + & - & - & - & - & + & $\|$ & 35 \\
\hline Rubus fruticosus agg. & $C$ & 1 & - & - & - & - & - & - & - & - & - & - & - & - & - & + & - & - & - & - & - & +-1 & I & 10 \\
\hline \multirow[t]{4}{*}{ Sambucus nigra } & $\mathrm{A} 2$ & - & - & - & - & - & - & - & - & - & - & - & - & - & - & - & 1 & - & - & - & - & 1 & I & 5 \\
\hline & B & - & - & - & - & - & 1 & - & + & - & - & + & 1 & - & 2 & - & + & - & + & + & - & +-2 & $\|$ & 40 \\
\hline & $C$ & - & - & + & + & + & + & - & - & - & + & - & + & - & + & + & + & + & + & - & + & + & III & 60 \\
\hline & $S$ & & & & & & & & & & & & & & & & & & & & & & IV & 75 \\
\hline Urtica dioica & $C$ & - & - & + & + & - & - & - & - & - & - & + & - & + & 1 & + & 2 & - & + & + & - & +-2 & III & 45 \\
\hline \multicolumn{25}{|c|}{ Adventiva (incl. Culta, Subspontanea \& Indigena) } \\
\hline
\end{tabular}

Erigeron annuus

Other

Arctium sp.

$$
\text { C }
$$




\begin{tabular}{|c|c|c|c|c|c|c|c|c|c|c|}
\hline & 1 & 2 & 3 & 4 & 5 & 6 & 7 & 8 & 9 & 10 \\
\hline Year, first relevé & 2006 & 2006 & 2006 & 2006 & 2006 & 2006 & 2006 & 2006 & 2006 & 2006 \\
\hline Date of first relevé & 10.05 & 08.13 & 10.05 & 08.10 & 08.10 & 08.15 & 08.15 & 08.20 & 08.20 & 08.18 \\
\hline Year, second relevé & 2007 & 2007 & 2007 & 2007 & 2007 & 2007 & 2007 & 2007 & 2007 & 2007 \\
\hline Date of second relevé & 03.17 & 03.25 & 03.17 & 03.05 & 03.06 & 03.06 & 03.06 & 03.05 & 03.05 & 03.07 \\
\hline Altitude $m$ a.s.l. & 325 & 340 & 305 & 315 & 350 & 325 & 330 & 330 & 325 & 365 \\
\hline Exposition & NE & $\mathrm{E}$ & NE & $\mathrm{E}$ & W & SE & $\mathrm{N}$ & $\mathrm{N}, \mathrm{NW}$ & W & $S$ \\
\hline Declination & 30 & 28 & 20 & 15 & 35 & 28 & 15 & 20 & 25 & 28 \\
\hline Upper canopy, cover (\%) & 70 & 75 & 65 & 60 & 65 & 60 & 75 & 80 & 80 & 70 \\
\hline Lower canopy, cover (\%) & 10 & 5 & 5 & 10 & 5 & 20 & - & 10 & 5 & 5 \\
\hline Shrub layer, cover (\%) & 0.1 & 15 & 4 & 15 & 20 & 5 & 3 & 5 & 5 & - \\
\hline Herb layer, cover (\%) & 90 & 70 & 95 & 100 & 100 & 100 & 95 & 100 & 100 & 100 \\
\hline Upper canopy, height (m) & 27 & 27 & 25 & 27 & 26 & 25 & 26 & 25 & 23 & 26 \\
\hline Lower canopy, height $(\mathrm{m})$ & 12 & 17 & 13 & 12 & 8 & 12 & - & 14 & 13 & 10 \\
\hline Shrub layer, height $(\mathrm{cm})$ & 140 & 170 & 150 & 200 & 450 & 200 & 150 & 150 & 150 & - \\
\hline \multirow[t]{2}{*}{ Herb layer, height (cm) } & 70 & 40 & 45 & 60 & 50 & 40 & 40 & 40 & 40 & 60 \\
\hline & 11 & 12 & 13 & 14 & 15 & 16 & 17 & 18 & 19 & 20 \\
\hline Year, first relevé & 2007 & 2006 & 2006 & 2007 & 2007 & 2008 & 2006 & 2006 & 2006 & 2006 \\
\hline Date of first relevé & 03.15 & 08.06 & 08.10 & 03.22 & 03.25 & 06.17 & 09.05 & 08.17 & 09.25 & 08.20 \\
\hline Year, second relevé & 2007 & 2007 & 2007 & 2007 & 2007 & 2009 & 2007 & 2007 & 2007 & 2007 \\
\hline Date of second relevé & 10.03 & 03.11 & 03.05 & 10.03 & 10.03 & 04.03 & 03.16 & 03.06 & 03.11 & 03.05 \\
\hline Altitude m a.s.l. & 295 & 350 & 325 & 355 & 360 & 475 & 350 & 335 & 345 & 315 \\
\hline Exposition & $W_{1}-$ & $\mathrm{N}$ & w & $\mathrm{N}_{1}-$ & $N, W_{1}-$ & $E$ & Var. & Var. & $\mathrm{N},-$ & $\mathrm{N}$ \\
\hline Declination & 20 & 15 & 28 & 35 & 40,30 & 30 & 30 & 20 & 30 & 30 \\
\hline Upper canopy, cover (\%) & 75 & 80 & 60 & 55 & 70 & 55 & 90 & 55 & 85 & 55 \\
\hline Lower canopy, cover (\%) & - & 5 & 3 & 10 & - & 5 & 5 & 5 & - & 4 \\
\hline Shrub layer, cover (\%) & 15 & 60 & 40 & 10 & 15 & 15 & - & 0.5 & 50 & 15 \\
\hline Herb layer, cover (\%) & 90 & 80 & 100 & 90 & 100 & 90 & 60 & 100 & 75 & 100 \\
\hline Upper canopy, height (m) & 23 & 30 & 25 & 27 & 30 & 25 & 25 & 30 & 28 & 25 \\
\hline Lower canopy, height (m) & - & 10 & 10 & 15 & - & 10 & 15 & 10 & - & 7 \\
\hline Shrub layer, height (cm) & 200 & 200 & 500 & 400 & 200 & 200 & - & 150 & 250 & 500 \\
\hline Herb layer, height (cm) & 65 & 45 & 60 & 50 & 70 & 50 & 25 & 50 & 45 & 50 \\
\hline Plot size $\left(m^{2}\right)$ & 400 & 400 & 400 & 400 & 400 & 400 & 400 & 400 & 400 & 400 \\
\hline
\end{tabular}

Locality: 1, 3, 17: Orfü “Vásáros út”; 2, 10-12, 19: Orfü “Cigány-föld”; 4, 13, 18: Orfü “Szuadó”; 5-9, 20: Orfu” “Száraz kút-pihenő”; 14: Pécs “Zsidóvölgy"; 15: Pécs "Lóri”; 16: Pécs "Lyukas-hárs"

Relevés 1-5, 10-20 made by Bátori, Z. (ined.); 6-9 made by Bátori, Z., Erdős, L. (ined.) 
Tab. 3: Synoptic table of the vegetation of the study area with fidelity values ( $\Phi$ x 100) and diagnostic species.

\begin{tabular}{|c|c|c|c|c|c|c|}
\hline & $\begin{array}{c}\text { I. } \\
\text { PmQd }\end{array}$ & $\begin{array}{c}\text { II. } \\
\text { TtFo }\end{array}$ & $\begin{array}{l}\text { III. } \\
\text { AtC }\end{array}$ & $\begin{array}{c}\text { V. } \\
\text { Dolines }\end{array}$ & $\begin{array}{l}\text { VI. } \\
\text { SaA }\end{array}$ & $\begin{array}{l}\text { VII. } \\
\text { CpA }\end{array}$ \\
\hline Festuca heterophylla & 91.4 & --- & --- & --- & --- & --- \\
\hline Lathyrus niger & 91.4 & --- & --- & --- & --- & --- \\
\hline Clinopodium vulgare & 78.1 & --- & --- & --- & --- & --- \\
\hline Tanacetum corymbosum & 76.5 & --- & --- & --- & --- & --- \\
\hline Sedum telephium subsp. maximum & 71.2 & --- & --- & --- & --- & --- \\
\hline Prunus spinosa & 70.7 & --- & --- & --- & --- & --- \\
\hline Trifolium alpestre & 68.0 & --- & --- & --- & --- & --- \\
\hline Silene viridiflora & 67.7 & --- & --- & --- & --- & --- \\
\hline Potentilla micrantha & 64.2 & 32.7 & --- & --- & --- & --- \\
\hline Euphorbia cyparissias & 63.9 & --- & --- & --- & --- & --- \\
\hline Luzula forsteri & 61.1 & --- & --- & --- & --- & --- \\
\hline Veronica officinalis & 60.4 & --- & --- & --- & --- & --- \\
\hline Lactuca quercina & 60.0 & --- & --- & --- & --- & --- \\
\hline Hieracium sabaudum & 57.4 & --- & --- & --- & --- & --- \\
\hline Poa nemoralis & 56.9 & --- & --- & --- & -- & --- \\
\hline Astragalus glycyphyllos & 56.3 & --- & --- & --- & --- & --- \\
\hline Brachypodium rupestre & 56.3 & --- & --- & --- & --- & --- \\
\hline Lychnis coronaria & 56.3 & --- & --- & --- & --- & --- \\
\hline Poapratensis & 56.3 & --- & --- & --- & --- & --- \\
\hline Trifolium rubens & 56.3 & --- & --- & --- & --- & --- \\
\hline Vincetoxicum officinale & 53.4 & --- & --- & --- & --- & --- \\
\hline Pyrus pyraster & 52.7 & --- & 31.3 & --- & --- & --- \\
\hline Chamaecytisus supinus & 51.9 & --- & --- & --- & --- & --- \\
\hline Securigera varia & 51.9 & --- & --- & --- & --- & --- \\
\hline Galium schultesii & 51.9 & --- & --- & --- & --- & --- \\
\hline Lysimachia punctata & 51.9 & --- & --- & --- & --- & --- \\
\hline Serratula tinctoria & 51.9 & --- & --- & --- & --- & --- \\
\hline Fallopia dumetorum & 51.5 & 37.5 & --- & --- & --- & --- \\
\hline Carex flacca & 47.2 & --- & --- & --- & --- & --- \\
\hline Euphorbia epithymoides & 47.2 & --- & --- & --- & --- & --- \\
\hline Genista ovata subsp. nervata & 47.2 & --- & --- & --- & --- & --- \\
\hline Rosa gallica & 47.2 & --- & --- & --- & --- & --- \\
\hline Vicia tetrasperma & 47.2 & --- & --- & --- & --- & --- \\
\hline Convallaria majalis & 47.1 & --- & --- & --- & --- & --- \\
\hline Carex divulsa & 45.5 & --- & --- & --- & --- & --- \\
\hline Galium mollugo & 45.1 & 32.9 & --- & --- & --- & --- \\
\hline Arabis turrita & --- & 89.6 & --- & --- & --- & --- \\
\hline Calamintha sylvatica subsp. sylvatica & --- & 60.7 & --- & --- & --- & --- \\
\hline Buglossoides purpureo-coerulea & --- & 53.4 & --- & --- & --- & --- \\
\hline Cystopteris fragilis & --- & 53.1 & --- & --- & --- & --- \\
\hline Tilia platyphyllos & --- & 46.7 & --- & --- & --- & --- \\
\hline Campanula trachelium & --- & 46.2 & --- & --- & --- & --- \\
\hline Viola odorata & --- & 46.2 & --- & --- & --- & --- \\
\hline Campanula rapunculoides & 37.2 & 45.5 & --- & --- & --- & --- \\
\hline Asperula taurina & --- & --- & 50.1 & --- & --- & --- \\
\hline Scutellaria altissima & --- & --- & 47.8 & --- & --- & --- \\
\hline Heracleum sphondylium & --- & --- & 46.8 & --- & --- & --- \\
\hline Dryopteris carthusiana & --- & --- & --- & 64.2 & --- & --- \\
\hline Athyrium filix-femina & --- & --- & --- & 57.4 & --- & --- \\
\hline Atropa bella-donna & --- & --- & --- & 50.1 & --- & --- \\
\hline Paris quadrifolia & --- & --- & --- & 46.8 & --- & --- \\
\hline
\end{tabular}




\begin{tabular}{|c|c|c|c|c|c|c|}
\hline & $\begin{array}{c}\text { I. } \\
\text { PmQd }\end{array}$ & $\begin{array}{c}\text { II. } \\
\text { TtFo }\end{array}$ & $\begin{array}{l}\text { III. } \\
\text { AtC }\end{array}$ & $\begin{array}{c}\text { V. } \\
\text { Dolines }\end{array}$ & $\begin{array}{l}\text { VI. } \\
\text { SaA }\end{array}$ & $\begin{array}{l}\text { VII. } \\
\text { CpA }\end{array}$ \\
\hline Asplenium scolopendrium & --- & --- & --- & --- & 77.9 & --- \\
\hline Lunaria rediviva & --- & --- & --- & --- & 68.0 & --- \\
\hline Cardamine amara & --- & --- & --- & --- & 64.2 & --- \\
\hline Polystichum aculeatum & --- & --- & --- & 37.5 & 56.2 & --- \\
\hline Silene dioica & --- & 33.6 & --- & --- & 51.7 & --- \\
\hline Robinia pseudo-acacia & --- & --- & --- & --- & 51.5 & --- \\
\hline Oxalis acetosella & --- & --- & --- & --- & 46.1 & --- \\
\hline Alnus glutinosa & --- & --- & --- & --- & --- & 94.4 \\
\hline Persicaria mitis & --- & --- & --- & --- & --- & 91.4 \\
\hline Calystegia sepium & --- & --- & --- & --- & --- & 84.9 \\
\hline Salix fragilis & --- & --- & --- & --- & --- & 79.9 \\
\hline Equisetum arvense & --- & --- & --- & --- & --- & 78.2 \\
\hline Rubus caesius & --- & --- & --- & --- & --- & 75.7 \\
\hline Myosoton aquaticum & --- & --- & --- & --- & --- & 74.8 \\
\hline Poa trivialis & --- & --- & --- & --- & --- & 71.6 \\
\hline Ranunculus repens & --- & --- & --- & --- & 37.0 & 71.3 \\
\hline Eupatorium cannabinum & --- & --- & --- & --- & --- & 70.7 \\
\hline Lycopus europaeus & --- & --- & --- & --- & --- & 70.7 \\
\hline Solanum dulcamara & --- & --- & --- & --- & --- & 69.6 \\
\hline Angelica sylvestris & --- & --- & --- & --- & --- & 68.0 \\
\hline Festuca gigantea & --- & --- & --- & --- & --- & 66.3 \\
\hline Petasites hybridus & --- & --- & --- & --- & --- & 64.3 \\
\hline Symphytum officinale & --- & --- & --- & --- & --- & 64.3 \\
\hline Equisetum telmateia & --- & --- & --- & --- & --- & 64.2 \\
\hline Knautia drymeia & --- & --- & --- & --- & --- & 64.2 \\
\hline Arctium minus & --- & --- & --- & --- & --- & 62.2 \\
\hline Berula erecta & --- & --- & --- & --- & --- & 60.4 \\
\hline Humulus lupulus & --- & --- & --- & --- & --- & 60.4 \\
\hline Aethusa cynapium & --- & --- & --- & --- & --- & 59.0 \\
\hline Ranunculus lanuginosus & --- & --- & --- & --- & 39.1 & 57.4 \\
\hline Lythrum salicaria & --- & --- & --- & --- & --- & 56.3 \\
\hline Galeopsis speciosa & --- & --- & --- & --- & --- & 55.1 \\
\hline Carex remota & --- & --- & --- & --- & 38.0 & 53.7 \\
\hline Adoxa moschatellina & --- & --- & --- & --- & --- & 53.1 \\
\hline Epilobium lanceolatum & --- & --- & --- & --- & --- & 51.9 \\
\hline Carex pendula & --- & --- & --- & --- & --- & 51.6 \\
\hline Cornus sanguinea & --- & --- & --- & --- & --- & 48.4 \\
\hline Galium aparine & --- & --- & --- & --- & --- & 48.2 \\
\hline Urtica dioica & --- & --- & --- & --- & 31.0 & 47.7 \\
\hline Veronica beccabunga & --- & --- & --- & --- & --- & 46.8 \\
\hline Corylus avellana & --- & --- & --- & --- & --- & 46.2 \\
\hline Total number of species (in the 20 relevés) & 200 & 174 & 154 & 114 & 174 & 215 \\
\hline Number of diagnostic species & 36 & 8 & 3 & 4 & 7 & 34 \\
\hline Proportion of diagnostic species (\%) & 18 & 5 & 2 & 4 & 4 & 16 \\
\hline
\end{tabular}

Abbreviations:

PmQd: Potentillo micranthae-Quercetum dalechampii; TtFo: Tilio tomentosae-Fraxinetum orni; AtC: Asperulo taurinae-Carpinetum; HoF: Helleboro odori-Fagetum; SaA: Scutellario altissimae-Aceretum; CpA: Carici pendulae-Alnetum; Var.: variable, A1: upper canopy layer, A2: lower canopy layer, B: shrub layer, C: herb layer, S: sum. 
The use of $\mathrm{N}$ values (Tab. 1) with binary data shows that the maximum is at $\mathrm{N} 4$ (plants of submesotrophic habitats) and N5 (plants of mesotophic habitats) in turkey oak-sessile oak forests, at N5 in rock forests, oakhornbeam forests and beech forests, at N7 (plants of soils rich in mineral nitrogen) in dolines, ravine forests and alder forests. The proportion of N5 species is considerable in all of the units, while N6 (plants of moderately nutrient rich habitats) and N7 species show similarly important values except for the turkey oak-sessile oak forests. When using the weighted data, the maximum is at N4 in the case of turkey oak-sessile oak forests, while concerning all the other units the maximum is found at higher values than the binary data.

In the case of $\mathrm{T}$ indicator values, the proportion of T5 (montane mesophilous broad-leaved forest belt) or T6 (submontane broad-leaved forest belt) species is the highest in the vegetation units, while the $\mathrm{R}$ indicator values show the maximum at R6 (mostly on neutral soils but also in acidic and basic ones, generally widely tolerant, more or less indifferent plants) or R7 (basifrequent plants, mostly on basic soils). According to the weighted data, the rate of R8 (basiphilous plants) in rock forests and R5 (plants of slightly acid soils) in turkey oak-sessile oak forests is also considerable.

\section{IMPORTANCE OF THE VEGETATION IN NATURE CONSERVATION}

The bottom of the dolines in Western Mecsek is not influenced much by human activity. Besides their vegetation, their near-natural state is shown by the presence of the fallen, mossy and decayed trees. Several protected species were detected in the lower part of the dolines. These are: Aconitum vulparia, Aremonia agrimonoides, Dryopteris affinis, Dryopteris carthusiana, Dryopteris dilatata, cf. Dryopteris expansa, Helleborus odorus, Hepatica nobilis, Galanthus nivalis, Lilium martagon, Polystichum aculeatum, Polystichum setiferum, Ruscus aculeatus, Ruscus hypoglossum, Stachys alpina, Tamus communis. Dryopteris affinis, cf. Dryopteris expansa and the relict Stachys alpina are rarities not only in the dolines but also in the whole country. Dryopteris affinis - a new species of the Mecsek Mts. - was recently discovered in the dolines and valleys of Western Mecsek (Bátori et al. 2006). Some other protected plants (Aruncus dioicus, Asperula taurina, Asplenium scolopendrium, Carex strigosa, Chaerophyllum aureum, Epipactis helleborine, Iris graminea, Lathyrus venetus, Lunaria rediviva, Neottia nidus-avis, Primula vulgaris, Silene dioica) occur also in the slopes and edges of these depressions.

\section{DISCUSSION AND CONCLUSION}

The deep valleys and the karst depressions contribute to the geomorphological and vegetation diversity of the landscape of the Western Mecsek. In this study, the vegetation and habitat conditions were investigated in the lower part of the dolines by the Braun-Blanquet method.

The multivariate analysis shows remarkable similarities among the relevés made in the dolines in Western Mecsek. Considering the species composition, the vegetation of the dolines resembles mainly the beechwoods and ravine forests.

The canopy layer of the dolines resembles that of the local ravine forests, in which Acer pseudoplatanus, Carpinus betulus, Fagus sylvatica, Fraxinus excelsior, or Tilia tomentosa are dominant (Kevey 1993, 1997). In contrary, the species composition of the herb layer of the dolines is markedly different. Many Fagetalia, Querco-Fagea and Tilio-Acerion elements are missing from the dolines that appear in the ravine forests. The vegetation of the dolines differs also from the beech forests of the Mecsek Mts. Several fern species and wet woodland species are missing from the beech forests that are typical in dolines.
Athyrium filix-femina, Atropa bella-donna, Dryopteris carthusiana and Paris quadrifolia are the most important differential species of the dolines.

Hoyk (1999a, b, 2002) and Hoyk and Keveiné Bárány (2000) studied the habitat conditions and the vegetation of the karst of Western Mecsek with soil analyses and ecological indicator values. They found that the majority of the species indicate a deciduous forest climate, together with a sub-mediterranean deciduous forest climate, and a mediterranean, atlantic evergreen forest climate. According to moisture supply, fresh, moderately fresh, and moderately wet conditions are indicated by most of the plants, while the distribution of soil reaction values shows near neutral and moderately calciphilous conditions. Our study revealed that the plant communities characteristic of the karst surface of Western Mecsek are arranged along a moisture and nutrient gradient. Turkey oak-sessile oak forests grow in the driest habitats with the highest rate of W3, W4 and W5 species in the indicator spectra. Rock forests and oak-hornbeam forests prefer moister habitats and, along the gradient, they are followed by beech forests, dolines and ravine forests. Al- 
der forests occur in the moistest habitats with the highest proportion of W7, W8 and W9 species in the indicator spectra. We can see a very similar arrangement between the habitats with the highest (alder forests) and the lowest (turkey oak-sessile oak forests) nitrogen supply. In this system, the habitat conditions of the dolines are similar to those of the beech forests and the local ravine forests: fresh and relatively rich in nutrients.

Due to the special funnel and bowl shape of the dolines, thermal inversion is evident, which results in sudden changes in the vegetation. This phenomenon causes the inversion of the zones of pine forests, dwarf pine thickets and alpine grasslands in the dolines of South-Eastern Alps (Borhidi 2002), or the separation of the fresh Festuco ovinae-Nardetum from the dry Festucetum sulcatae in the dolines in Bükk Mountain (North Hungary; Bacsó \& Zólyomi 1934). Some plant associations of the Slovenian Karst (Asaro-Carpinetum betuli, Ornithogalo pyrenaici-Carpinetum) also occur in the bottom of the dolines that are cooler and more humid than their environment (Kranjc 1997). Because of the climate-balancing effect of the forests, this phenomenon is not so striking in Mecsek Mts. (Lehmann 1970). In the karst, which is dominated by oak-hornbeam and beech forests, effects of the thermal inversion are the most spectacular where beech forests follow turkey oak-sessile oak forests and oak-hornbeam forests on the lower part of the doline slopes. The described vegetation type in the lower part of these depressions is developed by edafic factors, mainly by increased soil humidity.

Considering the vegetation texture and species composition, the vegetation of the dolines in Western Mecsek resembles mainly the local beechwoods and ravine forests. This vegetation type occurs only in small patches, its stands are disjunct and isolated fragments in the matrix of Illyrian beech and oak-hornbeam woods. Distinction of the vegetation of the dolines as a separate association is not supported by the analyses.

\section{ACKNOWLEDGEMENT}

The Authors are indebted to the Department of Plant Taxonomy and Geobotany of the University of Pécs, and the Department of Ecology of the University of Szeged for their indispensable help.

\section{REFERENCES}

Atalay, I., 2006: The Effects of Mountainous Areas on Biodiversity: A Case Study from the Northern Anatolian Mountains and the Taurus Mountains.- In: Proceedings of the $8^{\text {th }}$ International Symposium on High Mountain Remote Sensing Cartography, 21 $1^{\text {th }}$ $27^{\text {th }}$ March 2005, La Paz, Grazer Schriften der Geographie und Raumforschung, 41, 17-26, Graz.

Bacsó, N. \& B. Zólyomi, 1934: Mikroklíma és növényzet a Bükk-fennsíkon.- Az Időjárás, 38, 9-10, 177-196.

Bárány, I., 1967: Der Einfluss des Niveauunterschiedes und der Exposition auf die Lufttemperatur in einer Doline im Bükk-Gebirge.- Acta Climatologica Univ. Szegediensis, 7, 1-4, 85-109.

Bárány-Kevei, I., 1999: Microclimate of karstic dolines.Acta Climatologica Univ. Szegediensis, 32-33, 19 27.

Bartha, A., 1933: Szakosztály ügyek - Dracocephalum ruyschiana a Bükk hegységben.- Botanikai Közlemények, 30, 1-4, 114.
Bátori, Z., Baráth, K. \& J. Csiky, 2006: A Dryopteris affinis (Löwe) Fras.-Jenk. előfordulása a Mecsekben.Flora Pannonica, 4, 3-8.

Beck v. Mannagetta, G., 1906: Die Umkehrung der Pflanzenregionen in den Dolinen des Karstes.- Sitzungsber K Akad Wiss Wien Math ntw Klasse, 65, 3-4.

Borhidi, A., 1993: A magyar flóra szociális magatartás típusai, természetességi és relatív ökológiai értékszámai.- A Környezetvédelmi és Területfejlesztési Minisztérium Természetvédelmi Hivatala és a Janus Pannonius Tudományegyetem Kiadványa, pp. 93, Pécs.

Borhidi, A., 2002: Gaia zöld ruhája.- Magyar Tudományos Akadémia kiadványa, pp. 331, Budapest.

Borhidi, A., 2003: Magyarország növénytársulásai.- Akadémiai Kiadó, pp. 610, Budapest.

Boros, Á., 1935: A Szilicei és Barkai Jégbarlangok növényzete.- Botanikai Közlemények, 32, 1-6, 104-114. 
Boros, J. \& I. Bárány, 1975: Néhány adat egy bükki töbör keleti és nyugati lejtőjének fölmelegedéséhez.- Az Időjárás, 79, 297-300.

Braun-Blanquet, J., 1928: Pflanzensociologie.- Julius Springer Verlag, pp. 330, Berlin.

Brullo, S. \& G. Giusso del Galdo, 2001: Astracantha dolinicola (Fabaceae): a new species from Crete.- Nord. J. Bot., 21, 475-480.

Budai, J., 1913: Újabb adatok a Bükk hegység és dombvidéke flórájához.- Magyar Botanikai Lapok, 12, 315327.

Chytrý, M., Tichý, L., Holt, J. \& Z. Botta-Dukát, 2002: Determination of diagnostic species with statistical fidelity measures.- Journal of Vegetation Science, 13, 79-90.

Egli, B., Gerstberger, P., Greuter, W. \& H. Risse, 1990: Horstrissea dolinicola, a new genus and species of umbels (Umbelliferae, Apiaceae) from Kriti (Greece).- Willdenowia, 19, 389-399.

Egli, B. R., 1991: The special flora, ecological and edaphic conditions of dolines in the mountain of Crete.Botanika Chronika, 10, 325-335.

Favretto, D. \& L. Poldini, 1985: The vegetation in the dolinas of the karst region near Trieste (Italy).- Studia Geobotanica, 5, 5-18.

Futó, J., 1962: Mikroklimatikus mérések a Nagymezőn.Földrajzi Értesítő, 11, 487-498.

Geiger, R., 1950: Das Klima der bodennahen Luftschicht. Ein Lehrbuch der Mikroklimatologie.- Die Wissenschaft, 4. Auflage, pp. 460, Braunschweig.

Grom, S., 1959: Mahovna flora naših jam.- Naše jame, 1, 17-19.

Hill, M. O. \& H. G. Gauch, 1980: Detrended Correspondence Analysis: An improved ordination technique.- Vegetatio 42, 47-58.

Horvát, A. O., 1956: Mecseki tölgyesek erdőtípusai.- Janus Pannonius Múzeum Évkönyve, 131-148.

Horvát, A. O., 1958: Mecseki gyertyános-tölgyesek erdőtípusai.- Janus Pannonius Múzeum Évkönyve, 137-154.

Horvát, A. O., 1959: A mecseki bükkösök (Fagetum silvaticae mecsekense) erdőtípusai.- Janus Pannonius Múzeum Évkönyve, 31-48.

Horvát, A. O., 1972: Die Vegetation des Mecsekgebirges und seiner Umgebung.- Akadémiai Kiadó, pp. 376, Budapest.

Horvat, I., 1953: Vegetacija ponikava.- Geografski glasnik, 14-15, 1-25.

Hoyk, E., 1999a: Geoecological studies on the karstic surfaces of the planned protected area in Western Mecsek, South Hungary.- Acta Carsologica, 28, 2, 91-102.
Hoyk, E., 1999b: Investigation of the vegetation and soil in the dolinas of Western Mecsek Mountains, South Hungary.- Acta Carsologica, 28, 1, 105-114.

Hoyk, E. \& I. Keveiné Bárány., 2000: Tájökológiai szempontú vegetációelemzés a nyugat-mecseki karszton.- Karsztfejlődés, 5, 47-55.

Hoyk, E., 2002: Természetvédelmi szempontú geoökológiai értékelés a tervezett Nyugat-Mecsek Tájvédelmi Körzet területén.- PhD Thesis, Szegedi Tudományegyetem, pp. 112 .

Jakucs, L., 1971: A karsztok morfogenetikája. A karsztfejlődés varienciái.- Akadémiai Kiadó, pp. 310, Budapest.

Jakucs, L., 1980: A karszt biológiai produktum.- Földrajzi Közlemények, 28, 4, 331-339.

Jakucs, P., 1952: Újabb adatok a Tornense flórájához.Annales Biologicae Universitatum Hungariae, 2, 235-243.

Jakucs, P., 1961: Az Északi-középhegység keleti felének növényzete.- Földrajzi Értesítő, 10, 357-377.

Kevey, B., 1993: A Keleti-Mecsek szurdokerdei (Scutellario-Aceretum).- Folia Comloensis, 5, 29-53.

Kevey, B., 1997: A Nyugati-Mecsek szurdokerdei [Scutellario altissimae-Aceretum (Horvát A. O. 1958) Soó et Borhidi in Soó 1962]. Schluchtwälder des Westlichen Mecsek-Gebirges [Scutellario altissimae-Aceretum (Horvát A. O. 1958) Soó et BORHIDI in SOÓ 1962].- In: Borhidi A. \& L. Gy. Szabó (eds.) Studia Phytologica Jubilaria. Dissertationes in honorem jubilantis Adolf Olivér Horvát Doctor Academiae in annoversario nonagesimo nativitatis 1907-1997, pp. 75-99, Pécs.

Kevey, B. \& A. Borhidi, 1998: Top-forest (Aconito anthorae-Fraxinetum orni). A special ecotonal case in the phytosociological system (Mecsek Mts., South Hungary).- Acta Botanica Hungarica, 41, 1-4, 27-121.

Kevey, B. \& Á. Baranyi, 2002: A Nyugati-Mecsek égerligetei (Carici pendulae-Alnetum Borhidi \& Kevey 1996).- A Janus Pannonius Múzeum Évkönyve 1999-2000, 44-45, 5-24.

Kevey, B. \& A. Hirmann, 2002: „NS” számítógépes cönológiai programcsomag.- In: Aktuális flóra-és vegetációkutatások a Kárpát-medencében V., 2002. március 8-10. (Összefoglalók), 74, Pécs.

Kranjc, A. (ed.), 1997: Slovene Classical Karst-„Kras”.Inštitut za raziskovanje krasa ZRC SAZU, pp. 254, Postojna.

Lausi, D., 1964: Vorläufiger Überblick über die Vegetation der Triester Karstdolinen.- Acta Botanica Croatica Vol. Extraord., 65-71.

Lehmann, A., 1970: Tarvágás által okozott ökológiai változások az abaligeti karszton.- Pécsi Müszaki Szemle, $25,1,15-21$. 
Lovász, Gy., 1971: Adatok az Abaligeti-karszt geomorfológiai és hidrológiai jellemzéséhez.- Földrajzi Értesítő, 20, 3, 283-296.

Morschhauser, T., Márkus, A., Csiky, J., Vonyó, P., Gerely, L., Kóbor, I., Lőrinc, P., Mányoki, G., Vilisics, F, \& L. Lendvay, 2000: Vegetation map of the Jakab hill.Acta Biologica Debrecina, 11, 1, 280.

Morton, F., 1936: Relazione sulla vegetazione delle doline del Carso triestino. I. Communicazione.- Alpi Giulie, 37, 2, 57-70.

Pericin, C. \& H. Hürlimann, 2001: Beobachtungen zur vertikalen Verteilung der Moosarten in der Doline Sterna-Filaria im Karstgebiet von Buje/Buie in Istrien (Kroatien).- Bauhinia, 15, 91-96.

Pielou, E. C., 1984: The Interpretation of Ecological Data: A Primer on Classification and Ordination.- Wiley, pp. 288, New York.

Podani, J., 1994: Multivariate Data Analysis in Ecology and Systematics.- SPB Publishing, pp. 316, The Hague.

Polli, E., 2004: Aspetti speleovegetazionali della „Jama Dimnice”, 736 S („Grotta del Fumo”, 626 VG) nel Matarsko Podolje (Valsecca di Castelnuovo) di Markovščina (Carso Sloveno). Contributo alla conoscenza della speleoflora vascolare carsica.- Attie e Memorie della Commissione Grotte „E. Boegan”, 40, 63-83.

Polli, S., 1961: Il clima delle doline del Carso triestino.In: Atti del XVIII Congresso Geografico Italiano, Trieste 4-9 aprile 1961, 1-9, Trieste.

Polli, S., 1984: Guida naturalistica alla Conca di Percedol, Il clima (Carso triestino).- Villaggio del Fanciullo, 9-22.

Rónaki, L., 1972: A Ny-Mecseki karszt vízföldtani kutatásának újabb eredményei.- A Magyar Hidrológiai Társaság Pécsi Csoportjának Jubileumi Évkönyve 1952-1972, 121-146.

Salamon-Albert, É. \& T. Morschhauser, 2002: A zonalitási jelleg és az ökológiai indikátorérték spektrumok. In: Salamon-Albert, É. (ed.) Magyar botanikai kutatások az ezredfordulón, pp. 589-605, Pécs.

Sauli, G., 1972: Dati floristici e microclimatici di un pozzo naturale carsico.- Atti del Museo Civico di Storia Naturale di Trieste, 28, 1, 101-110.
Simon, T., 2000: A magyarországi edényes flóra határozója.- Nemzeti Tankönyvkiadó Rt., pp. 976, Budapest.

Søndergaard, P. \& B. Egli, 2006: Zelkova abelicea (Ulmaceae) in Crete: floristics, ecology, propagation and threats.- Willdenowia, 36 (Special Issue), 317-322.

Tan, K., Perdetzoglou, D. K. \& V. Roussis, 1997: Biebersteinia orphanidis (Geraniaceae) from southern Greece.- Annales Botanici Fennici, 34, 41-45.

Ter Braak, C. J. F, \& P. Šmilauer, 2002: CANOCO Reference Manual and CanoDraw for Windows User's Guide.- Software for Canonical Community Ordination (version 4.5). Microcomputer Power, pp. 500 , Ithaca NY.

Tichý, L., 2002: JUICE, software for vegetation classification.- Journal of Vegetation Science, 13, 451-453.

Tichý, L. \& M. Chytrý., 2006: Statistical determination of diagnostic species for site groups of unequal size.Journal of Vegetation Science, 17, 809-818.

Varga, Z., Sipos, J., Orci, M. K. \& I. Rácz., 2000: Félszáraz gyepek az Aggteleki-karszton: fitocönológiai viszonyok, egyenesszárnyú rovar-és lepkeegyüttesek.- In: Virágh K. \& Kun A. (eds.) Vegetáció és Dinamizmus. A 70 éves Fekete Gábort köszöntik tanítványai, barátai és munkatársai, pp. 195-238, Vácrátót.

Veress, M., 2004: A karszt.-BDF Természetföldrajzi tanszék, pp. 215, Szombathely.

Virók, V. \& R. Farkas, 2008: Új növényfaj a hazai edényes flórában: Haller-kövifoszlár (Cardaminopsis halleri (L.) Hayek).- Kitaibelia, 13, 1, 29-33.

Vojtkó, A., 1997: Új adatok a Tornai-karszt flórájához és vegetációjához.- Kitaibelia, 2, 2, 248-249.

Wagner, R., 1963: Der Tagesgang der Lufttemperatur einer Doline im Bükk-Gebirge.- Acta Climatologica Univ. Szegediensis, 2-3, 1-4, 49-79.

Yannitsaros, A. G., Constantinidis, T. A. \& D. D. Vassiliades, 1996: The rediscovery of Biebersteinia orphanidis Boiss. (Geraniaceae) in Greece.- Botanical Journal of the Linnean Society, 120, 239-242. 